\title{
Analgesic effect of paracetamol monotherapy vs. the combination of paracetamol/parecoxib vs. the combination of pethidine/paracetamol in patients undergoing thyroidectomy
}

Francesk Mulita ${ }^{1}$, Georgios-Ioannis Verras ${ }^{1}$, Fotios Iliopoulos ${ }^{1}$, Charalampos Kaplanis ${ }^{1}$, Elias Liolis ${ }^{2}$, Levan Tchabashvili ${ }^{1}$, Christos Tsilivigkos ${ }^{1}$, Ioannis Perdikaris ${ }^{1}$, Argyro Sgourou ${ }^{3}$, Adamantia Papachatzopoulou ${ }^{4}$, Ioannis Maroulis ${ }^{1}$

${ }^{1}$ Department of Surgery, General University Hospital of Patras, Greece

2Department of Internal Medicine, Division of Oncology, General University Hospital of Patras, Greece

${ }^{3}$ Biology Laboratory, School of Science and Technology, Hellenic Open University, Patras, Greece

${ }^{4}$ Laboratory of General Biology, Medical Faculty, University of Patras, Patras, Greece

\begin{abstract}
Introduction: The purpose of this study was to investigate the analgesic effect of 3 different regimens of combination analgesics administered to patients undergoing thyroidectomy.

Material and methods: A total of 152 patients undergoing total or subtotal thyroidectomy were enrolled. Patients allocated to group A received a combination of intravenous (IV) paracetamol and intramuscular (IM) pethidine, patients in group B received a combination of IV paracetamol and IV parecoxib, while patients in group $C$ received IV paracetamol monotherapy.

Results: The analgesic regimens of groups A and B were found to be of equivalent efficacy ( $p$-value $=1.000$ ). In contrast, patients in group $C$ (paracetamol monotherapy) had higher numerical rating scale scores, compared to both patients in groups A ( $p$-value $<0.001)$ and B ( $p$-value $<0.001)$.

Conclusions: The combinations of IV paracetamol with either IM pethidine or IV parecoxib are superior to IV paracetamol monotherapy in achieving pain control in patients undergoing thyroid surgery.
\end{abstract}

Key words: pethidine, parecoxib, postoperative pain, pain assessment, thyroidectomy.

\section{Introduction}

Thyroid disorders are among the most common endocrine diseases. A thyroid surgery may be necessary to treat these disorders $[1,2]$. Thyroidectomy is recommended for benign condition such as symptomatic large goitres and for the treatment of malignant disease of the thyroid gland [3]. Thyroidectomy has potential complications. The major postoperative complications are hypocalcaemia, wound infection, haematoma, and recurrent laryngeal nerve injury [4-6]. Hypoparathyroidism is the usual cause of hypocalcaemia and mostly occurs on the first and second days after surgery, and most physicians obtain serial serum calcium measurements after surgery to recognize and manage the low levels of calcium [7]. All patients undergoing surgery experience postoperative pain. Acute as well as chronic postoperative pain (CPOP) exists in varying degrees for every type of surgery [8]. Chronic postoperative pain is a poorly recognized potential outcome from surgery, which affects millions of patients every year, resulting in patient suffering and ensuing economic consequences. The intensity of acute postoperative pain is one of the risk factors of CPOP. Almost $37 \%$ of patients after thyroidectomy declare CPOP [9]. Parecoxib and paracetamol are non-opioid analgesics with a well-documented efficacy after different surgical procedures. The use of non-opioid analgesics can reduce opioidinduced side-effects [10, 11]. Non-steroidal anti-inflammatory drugs (NSAIDs) inhibit the enzymes cyclooxygenase (COX)-1 and -2. Only the inhibition of COX-2 is involved in analgesic, anti-inflammatory, and antipyretic effects of NSAIDs [12]. This clinical study was designed to contrast the analgesic efficacy of 3 analgesic regimens in the setting of thyroid surgery: paracetamol monotherapy vs. paracetamol combinations with either pethidine or parecoxib. Studies investigating the analgesic effects of combined pethidine and paracetamol have not been published so far.

\section{Material and methods}

\section{Patient selection}

This prospective, randomized trial was conducted in our institution. Ethical approval was obtained from

Corresponding author:

Francesk Mulita, MD, Department of Surgery, General University Hospital of Patras, Greece,

Submitted: 31.05 .2021

e-mail: oknarfmulita@hotmail.com 
the local Ethics Committee. Between February 2017 and May 2019, 152 patients undergoing elective total or subtotal thyroidectomy were enrolled in the study. All patients provided written informed consent. Inclusion criteria were age between 35 and 65 years, American Society of Anaesthesiologists physical status classification I or II, and diagnosis of nontoxic multinodular goitre that was scheduled to be treated by elective total or subtotal thyroidectomy. Preoperative evaluation for general anaesthesia was performed. Exclusion criteria were heart failure, liver failure, renal dysfunction, diabetes, severe bronchial asthma, neurological or psychiatric disease, history of chronic pain or opioid intake, difficulty in communication due to language barriers or intellectual disability, and history of adverse events after NSAIDs (paracetamol, parecoxib) or pethidine administration. The day before surgery, the patients gave informed written consent to the study. The day prior to surgery the patients were introduced to the numerical rating scale (NRS) for pain documentation.

\section{Study setting}

All participants were randomly assigned to each group before surgery, using a computer-generated random number generator and sequentially numbered opaque sealed envelopes. Patients in group A were randomized to receive IV paracetamol $1000 \mathrm{mg}$ every 8 hours and intramuscular (IM) pethidine $50 \mathrm{mg}$ every 6 hours. Patients in group B were randomized to receive a combination of intravenous (IV) paracetamol $1000 \mathrm{mg}$ every 8 hours and IV parecoxib $40 \mathrm{mg}$ every 12 hours. Finally, patients in group $C$ were randomized to receive IV paracetamol 1000 mg every 8 hours only. Patients who asked for more postoperative analgesics were excluded from this trial. All operations were conducted by the same group of surgeons and anaesthesiologists. General anaesthesia consisted of IV fentanyl $0.5-1.5 \mu \mathrm{g} / \mathrm{kg}$ and propofol. All patients received IV paracetamol $1000 \mathrm{mg}$, IV parecoxib $40 \mathrm{mg}$, and IM pethidine $50 \mathrm{mg}$ during the procedure.

\section{Postoperative pain assessment}

In general, the procedure following the surgery and placement of the skin sutures is the extubation of patients in the surgical room. Surgery information is recorded, such as surgery time, and analgesics used.

Following surgery, patients were transferred to the surgical ward. Patients were evaluated at the bedside at 45 minutes, 2 hours, 6 hours, 12 hours, and 24 hours after receiving the first analgesic dose from their allocated regimen. Patients' NRS pain ratings were recorded on postoperative monitoring charts. The scale ranges from 0 to 10 , where 0 means no pain and 10 corresponds to the maximum possible pain. Four commonly used pain intensity scales are the NRS, the visual analogue scales, the verbal rating scales, and the faces pain rating scales [13-15]. There is a consensus that NRSs have more validity and more strengths than other scales [14-21]. Another reason why NRS was chosen in this trial is the fact that the medical staff and researchers are more familiar with the use of this scale.

\section{Statistical analysis}

The data were collected with the pretested questionnaires and analysed using Stata 13 statistical software. Mean and standard deviations were determined for continuous variables. The analysis of pain scores was expressed as mean and 95\% confidence interval. The postoperative pain intensities measured by NRS within groups and between groups at each time interval were analysed using one-way repeat measured analysis of variance (ANOVA) and post hoc test-Bonferroni correlation. Normality of the data was tested using Shapiro-Wilk test for normality. The post hoc test was used to uncover specific differences between the 3 group means because the ANOVA test was significant. In order to use post hoc test-Bonferroni correlation, an appropriate sample size of 20 patients from each group was required for validity. $P$-values less than 0.05 were considered significant.

\section{Results}

We approached 169 patients and assessed them for eligibility; 17 did not meet our inclusion criteria as shown on the flowchart in Figure 1. A total of 152 patients, including 30 males and 122 females, were randomized into 3 groups: the paracetamol and pethidine group, the paracetamol and parecoxib group, and the paracetamol (monotherapy) group. After randomization, no participant was withdrawn from the trial (Fig. 1). The mean age of the patients in the paracetamol and pethidine group (group A) was $54.1 \pm 3.8$ years, and the mean duration time was $79.8 \pm 14.1$ in the group. The mean ages of the patients were $52.4 \pm 3.5$ years and $53.7 \pm 4.4$ years for the paracetamol/parecoxib group (group B) and paracetamol monotherapy group (group $\mathrm{C}$ ), respectively. In addition, the mean operation time for group B and group C was $81.9 \pm 7.4$ and $80.1 \pm 12$, respectively. The patients' baseline characteristics are shown in Table 1.

\section{Postoperative pain assessment}

The mean NRS for patients who were treated with IV paracetamol and IM pethidine (group A) were 4.92 at 45 minutes (0.75 hours), 4.07 at 2 hours, 2.66 at 6 hours, 1.81 at 12 hours, and 0.90 at 24 hours. The mean 


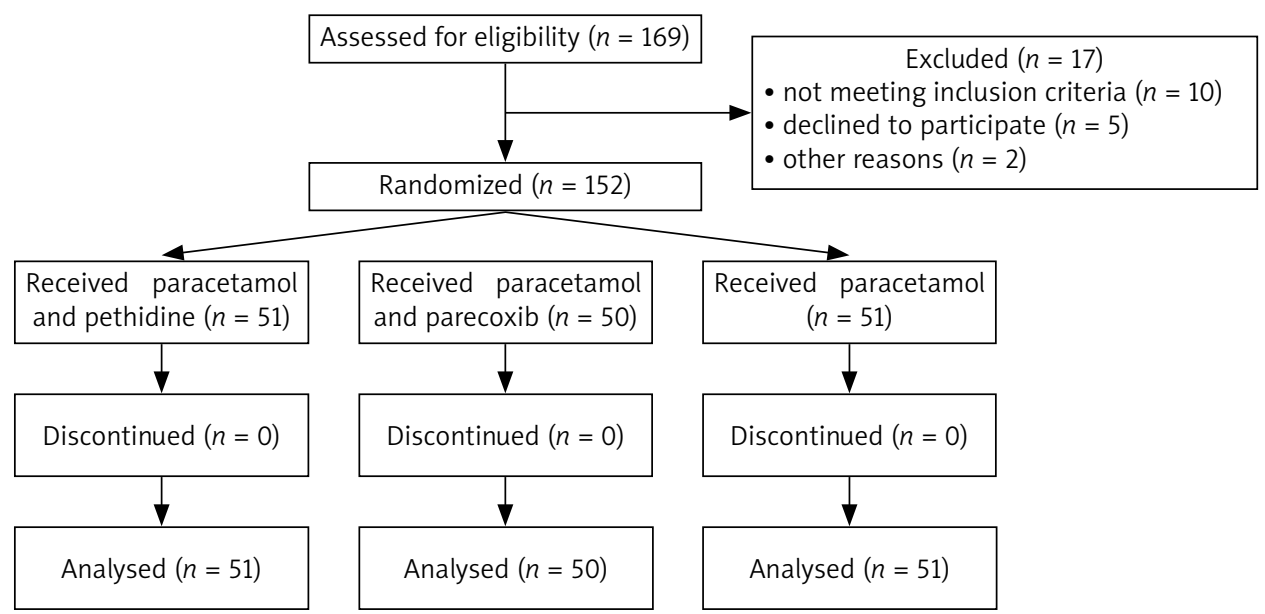

Fig. 1. Flowchart showing recruitment and randomization of participants

Table 1. Baseline characteristics of the study population

\begin{tabular}{lccc}
\hline Variable & $\begin{array}{c}\text { Group A - } \\
\text { paracetamol and pethidine }\end{array}$ & $\begin{array}{c}\text { Group B - } \\
\text { paracetamol and parecoxib }\end{array}$ & $\begin{array}{c}\text { Group C - } \\
\text { paracetamol } \\
\text { monotherapy }\end{array}$ \\
\hline Number of patients $(n=152)$ & 51 & 50 & 51 \\
\hline Males/females (30/122) & $10 / 41$ & $9 / 41$ & $11 / 40$ \\
\hline Age (Mean \pm SD) (years) & $54.1 \pm 3.8$ & $52.4 \pm 3.5$ & $53.7 \pm 4.4$ \\
\hline Operative time (Mean \pm SD) (minutes) & $79.8 \pm 14.1$ & $81.9 \pm 7.4$ & $80.1 \pm 12$ \\
\hline Dosage in 24 h & $\begin{array}{c}\text { Paracetamol } 1 \mathrm{~g} / 8 \mathrm{~h} \\
\text { Pethidine } 50 \mathrm{mg} / 6 \mathrm{~h}\end{array}$ & $\begin{array}{c}\text { Paracetamol } 1 \mathrm{~g} / 8 \mathrm{~h} \\
\text { Parecoxib 40 } \mathrm{mg} / 12 \mathrm{~h}\end{array}$ & Paracetamol $1 \mathrm{~g} / 8 \mathrm{~h}$ \\
\hline
\end{tabular}

SD - standard deviations

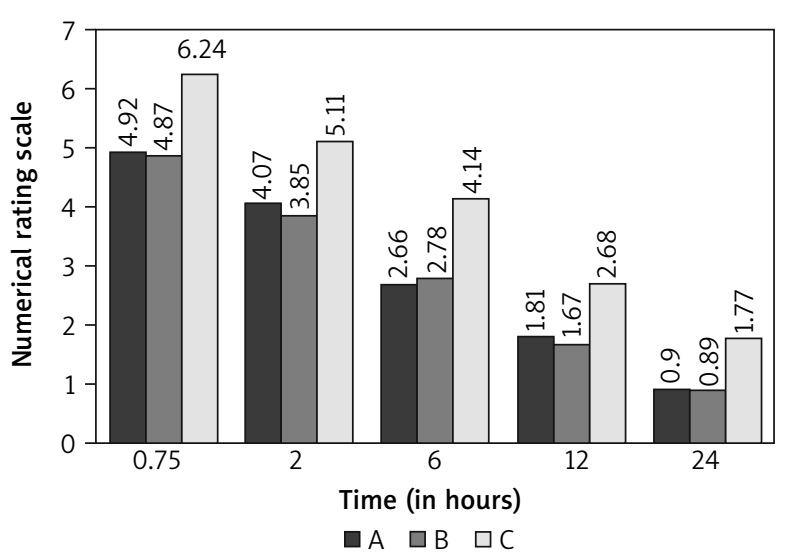

Fig 2. Mean numerical rating scale between the patients of group A (paracetamol and pethidine), group B (paracetamol and parecoxib), and group C (paracetamol - monotherapy) based on time

NRS for patients who were treated with IV paracetamol and IV parecoxib (group B) were 4.87 at 45 minutes (0.75 hours), 3.85 at 2 hours, 2.78 at 6 hours, 1.67 at 12 hours, and 0.89 at 24 hours, while the mean NRS for patients who were treated with only IV paracetamol (group C) were 6.24 at 45 minutes (0.75 hours), 5.11 at 2 hours, 4.14 at 6 hours, 2.68 at 12 hours, and 1.77 at 24 hours (Fig. 2). The NRS scores of group C (paracetamol monotherapy) were significantly higher than those of groups A (pethidine + paracetamol, $p<0.001$ ) and $B$ (paracetamol + parecoxib, $p<0.001$ ), while there was no significant difference between patients of group A and group $B(p=1.00)$.

\section{Discussion}

According to recent studies, there is no ideal postoperative analgesic treatment regimen for patients undergoing thyroid surgery. However, there are many studies that have found that nonopioid adjuncts decreased patients' need for postoperative opioids [22]. The reduction of opioid requirements using postoperative non-opioid analgesics in patients after surgery is very important in reducing sedation, impaired pulmonary function, and constipation [23]. In our study, the influence of paracetamol and its combination with parecoxib and pethidine on postoperative consumption in a randomized, controlled trial was investigated. Patients included in this analysis underwent a thyroid surgery under general anaesthesia using a standardized surgical and anaesthetic technique. The results of this randomized, prospective study suggest that the combination of postoperative analgesic treatment with paracetamol and parecoxib is equivalent to the combination of paracetamol and pethidine. Both combinations were found to be superior to paracetamol 
monotherapy in achieving pain control in patients with thyroidectomy and should therefore be preferred in this setting. Furthermore, because these 2 regimens of analgesics appear to have similar efficacy, the combination of paracetamol and parecoxib should be preferred over paracetamol and pethidine, to reduce opioid consumption and associated adverse events [24, 25]. Opioid-related adverse effects in surgical patients are associated with increased length of stay in hospital and total hospital costs. The use of opioid-sparing techniques can be cost-effective [26, 27]. In a large cohort study ( $n=37.031)$, postsurgical patients experiencing an opioid-related adverse effect had a 55\% longer hospital stay, $47 \%$ higher costs, $36 \%$ increased risk of readmission, and 3.4 times higher risk of inpatient mortality [28]. In a trial in patients undergoing thyroid surgery the combination of parecoxib and paracetamol was found not to be superior to each substance alone [23]. However, studies investigating the analgesic effect of combined pethidine/paracetamol and parecoxib/ paracetamol have not been published so far in patients undergoing thyroidectomy. Studies comparing the analgesic efficacy of paracetamol monotherapy vs. paracetamol combinations with either pethidine or parecoxib in patients undergoing laparoscopic cholecystectomy and open inguinal hernia repair have found the same results as our study $[29,30]$. One limitation of this study that should be considered is that we did not record data during mobilization, because pain scores were recorded only at rest. The pain rating at rest alone is not very helpful because it is the functional outcome that is of clinical interest. Evaluation of pain during movement is suggested for further study [31].

\section{Conclusions}

In conclusion, the combination of postoperative analgesic treatment of IV paracetamol and IV parecoxib IV is equivalent to the combination of IV paracetamol and $I M$ pethidine in patients undergoing thyroid surgery. Both combinations of postoperative analgesics outweigh the paracetamol monotherapy and should therefore be preferred in thyroidectomy. Furthermore, our study confirms the notion of a significant opioid-sparing effect of parecoxib in postoperative pain management after thyroidectomy.

\section{Disclosure}

The authors report no conflict of interest.

\section{References}

1. Sun GH, de Monner S, Davis MM. Epidemiological and economic trends in inpatient and outpatient thyroidectomy in the United States, 19962006. Thyroid 2013; 23: 727-733.
2. Mulita F, Anjum F. Thyroid adenoma. In: StatPearls [Internet]. Treasure Island (FL): StatPearls Publishing; 2020; PMID: 32965923.

3. Hegner CF. A history of thyroid surgery. Ann Surg 1932; 95: 481-492.

4. Reeve T, Thompson NW. Complications of thyroid surgery: how to avoid them, how to manage them, and observations on their possible effect on the whole patient. World J Surg 2000; 24: 971.

5. Rosato L, Avenia N, Bernante P, et al. Complications of thyroid surgery: analysis of a multicentric study on 14,934 patients operated on in Italy over 5 years. World J Surg 2004; 28: 271-276.

6. Christou N, Mathonnet M. Complications after total thyroidectomy. J Visc Surg 2013; 150: 249-256.

7. Chahardahmasumi E, Salehidoost R, Amini M, et al. Assessment of the early and late complication after thyroidectomy. Adv Biomed Res 2019; 8: 14 .

8. Wattier JM, Caïazzo R, Andrieu G, Kipnis E, Pattou F, Lebuffe G. Chronic post-thyroidectomy pain: Incidence, typology, and risk factors. Anaesth Crit Care Pain Med 2016; 35: 197-201.

9. Correll D. Chronic postoperative pain: recent findings in understanding and management. F1000Res 2017; 6: 1054.

10. Ng A, Parker J, Toogood L, Cotton BR, Smith G. Does the opioid-sparing effect of rectal diclofenac following total abdominal hysterectomy benefit the patient? Br J Anaesth 2002; 88: 714-716.

11. Iohom G, Walsh M, Higgins G, Shorten G. Effect of perioperative administration of dexketoprofen on opioid requirements and inflammatory response following elective hip arthroplasty. Br J Anaesth 2002; 88: 520-526.

12. Munsterhjelm E, Niemi TT, Ylikorkala O, Neuvonen PJ, Rosenberg PH. Influence on platelet aggregation of i.v. parecoxib and acetaminophen in healthy volunteers. Br J Anaesth 2006; 97: 226-231.

13. Hjermstad MJ, Gibbins J, Haugen DF, Caraceni A, Loge JH, Kaasa S. Pain assessment tools in palliative care: an urgent need for consensus. Palliative Med 2008; 22: 895-903.

14. Pathak A, Sharma S, Jensen MP. The utility and validity of pain intensity rating scales for use in developing countries. Pain Rep 2018; 3: e672.

15. Thong ISK, Jensen MP, Miró J, Tan G. The validity of pain intensity measures: what do the NRS, VAS, VRS, and FPS-R measure? Scand J Pain 2018; 18: 99-107.

16. Chanques G, Viel E, Constantin JM, et al. The measurement of pain in intensive care unit: comparison of 5 self-report intensity scales. Pain 2010; 151: 711-721.

17. Hjermstad MJ, Fayers PM, Haugen DF, et al. European palliative care research collaborative. studies comparing numerical rating scales, verbal rating scales, and visual analogue scales for assessment of pain intensity in adults: a systematic literature review. J Pain Symptom Manage 2011; 41: 1073-1093.

18. Jensen MP, Karoly P, Braver S. The measurement of clinical pain intensity: a comparison of six methods. Pain 1986; 27: 117-126.

19. Jensen MP, Karoly P, O'riordan EF, Bland F, Burns RS. The subjective experience of acute pain. An assessment of the utility of 10 indices. Clin J Pain 1989; 5: 153-160.

20. Sánchez-Rodríguez E, Miró J, Castarlenas E. A comparison of four selfreport scales of pain intensity in 6- to 8-year-old children. Pain 2012; 153: 1715-1719.

21. Von Korff M, Jensen MP, Karoly P. Assessing global pain severity by selfreport in clinical and health services research. Spine 2000; 25: 31403151.

22. Uhlmann RA, Reinhart HA, Postevka E, Snyder SK, Romero Arenas M. A review of postoperative pain management for thyroid and parathyroid surgery. J Surg Res 2019; 241: 107-111.

23. Gehling M, Arndt C, Eberhart LH, Koch T, Krüger T, Wulf H. Postoperative analgesia with parecoxib, acetaminophen, and the combination of both: a randomized, double-blind, placebo-controlled trial in patients undergoing thyroid surgery. Br J Anaesth 2010; 104: 761-767.

24. Nong L, Sun Y, Tian Y, Li H, Li H. Effects of parecoxib on morphine analgesia after gynecology tumor operation: a randomized trial of parecoxib used in postsurgical pain management. J Surg Res 2013; 183: 821-826.

25. Fu W, Yao J, Li Q et al. Efficacy and safety of parecoxib/phloroglucinol combination therapy versus parecoxib monotherapy for acute renal colic: a randomized, double-blind clinical trial. Cell Biochem Biophys 2014; 69: 157-161. 
26. Oderda GM, Gan TJ, Johnson BH, Robinson SB. Effect of opioid-related adverse events on outcomes in selected surgical patients. J Pain Palliat Care Pharmacother 2013; 27: 62-70.

27. Philip BK, Reese PR, Burch SP. The economic impact of opioids on postoperative pain management. J Clin Anesth 2002; 14: 354-364.

28. Kessler ER, Shah M, Gruschkus SK, Raju A. Cost and quality implications of opioid-based postsurgical pain control using administrative claims data from a large health system: opioid-related adverse events and their impact on clinical and economic outcomes. Pharmacotherapy 2013; 33: 383-391.

29. Mulita F, Karpetas G, Liolis E, Vailas M, Tchabashvili L, Maroulis I. Comparison of analgesic efficacy of acetaminophen monotherapy versus acetaminophen combinations with either pethidine or parecoxib in patients undergoing laparoscopic cholecystectomy: a randomized prospective study. Med Glas (Zenica) 2021; 18: 27-32.

30. Mulita F, Parchas N, Solou K, et al. Postoperative pain scores after open inguinal hernia repair: comparison of three postoperative analgesic regimens. Med Arch 2020; 74: 355-358.

31. Shen SJ, Peng PY, Chen HP, Lin JR, Lee MS, Yu HP. Analgesic effects of intra-articular bupivacaine/intravenous parecoxib combination therapy versus intravenous parecoxib monotherapy in patients receiving total knee arthroplasty: a randomized, double-blind trial. Biomed Res Int 2015; 2015: 450805. 